Case report

\title{
A novel compound heterozygous mutation in the POMK gene causing limb-girdle muscular dystrophy-dystroglycanopathy in a sib pair
}

\author{
Sonja Strang-Karlsson ${ }^{\mathrm{a}, \mathrm{b}, *}$, Katherine Johnson ${ }^{\mathrm{c}}$, Ana Töpf ${ }^{\mathrm{c}}$, Liwen $\mathrm{Xu}^{\mathrm{d}, \mathrm{e}}$, Monkol Lek ${ }^{\mathrm{d}, \mathrm{e}}$, \\ Daniel G. MacArthur ${ }^{\mathrm{d}, \mathrm{e}}$, Olivera Casar-Borota ${ }^{\mathrm{f}, \mathrm{g}}$, Maria Williams ${ }^{\mathrm{h}}$, Volker Straub ${ }^{\mathrm{c}}$, \\ Carina Wallgren-Pettersson ${ }^{\mathrm{a}}$ \\ ${ }^{a}$ The Folkhaelsan Department of Medical Genetics, The Folkhaelsan Institute of Genetics and the Department of Medical and Clinical Genetics, University \\ of Helsinki, Helsinki, Finland \\ ${ }^{\mathrm{b}}$ Children's Hospital, Helsinki University Hospital and University of Helsinki, Helsinki, Finland \\ ${ }^{\mathrm{c} J o h n}$ Walton Muscular Dystrophy Research Centre, Institute of Genetic Medicine, Newcastle University, Newcastle upon-Tyne, United Kingdom \\ ${ }^{\mathrm{d}}$ Analytic and Translational Genetics Unit, Massachusetts General Hospital, Boston, MA 02114, USA \\ ${ }^{\mathrm{e}}$ Program in Medical and Population Genetics, Broad Institute of Harvard and MIT, Cambridge, MA 02142, USA \\ ${ }^{\mathrm{f}}$ Department of Immunology, Genetics and Pathology, Uppsala University, Uppsala, Sweden \\ ${ }^{\mathrm{g}}$ Department of Clinical Pathology, Uppsala University Hospital, Uppsala, Sweden \\ $\mathrm{h}$ Åland Central Hospital, Mariehamn, Åland, Finland
}

Received 21 December 2017; received in revised form 5 April 2018; accepted 26 April 2018

\begin{abstract}
We describe two Finnish siblings in whom an incidentally detected elevated creatine kinase activity eventually led to a diagnosis of limb-girdle muscular dystrophy-dystroglycanopathy (Type C12; MDDGC12). When diagnosed at age 10 and 13 years, they were mildly affected with a slow or non-progressive disease course. The main symptoms comprised infrequent hip cramps triggered by flexion, neck cramps triggered by yawning, transient growing pains, calf hypertrophy and mild proximal muscle weakness. Their cognitive and motor developments were unremarkable and they were physically active. Whole-exome sequencing revealed compound heterozygous mutations, both of which were novel, in the protein O-mannosyl kinase (POMK) gene in both siblings; a missense mutation, p.Pro322Leu (c.965C > T), and a nonsense mutation, p.Arg46Ter $(\mathrm{c} .136 \mathrm{C}>\mathrm{T})$. The results were confirmed by Sanger sequencing, showing that the parents were heterozygous carriers of one mutation each. This report adds to the literature by providing phenotype and genotype data on this ultra-rare POMK-related dystroglycanopathy.
\end{abstract}

(C) 2018 Elsevier B.V. All rights reserved.

Keywords: Protein-O-mannosyl kinase; POMK; Dystroglycanopathy; Limb-girdle muscular dystrophy-dystroglycanopathy type 12 C; MDDGC12; LGMD12C; Muscular dystrophy.

\section{Introduction}

Dystroglycanopathies are a group of muscular dystrophies characterised by considerable clinical and genetic heterogeneity. They are caused by disruption in the interactions between the transmembrane protein dystroglycan and extracellular matrix components. These interactions are necessary for normal

\footnotetext{
* Corresponding author at: The Folkhaelsan Department of Medical Genetics, Topeliusgatan 20, 00250 Helsinki, Finland.

E-mail address: sonja.strang@helsinki.fi (S. Strang-Karlsson).
}

muscle and brain development [1]. In these interactions, glycosylation plays a crucial role. Most known dystroglycanopathy mutations are located in genes related to the glycosylation process rather than in the dystroglycan gene itself [1].

The POMK gene (MIM 615247), located on chromosome 8 p11, encodes the protein O-mannose kinase which is necessary for proper glycosylation and function of the dystroglycan complex [2]. Biallelic mutations in the POMK gene cause limb-girdle muscular dystrophy-dystroglycanopathy (type C12; MDDGC12) (MIM 616094). To our knowledge, two such families have been described; one in which two 
Jordanian sibs presented with limb-girdle muscular dystrophy (LGMD) and cognitive impairment, and another in which a child born to consanguineous parents presented with LGMD, mild learning difficulties and congenital mirror movements [1,3]. POMK mutations can also cause the more severe Walker-Warburg syndrome (Muscular dystrophydystroglycanopathy, congenital with brain and eye anomalies, type A12; MDDGA12) (MIM 615249), encompassing brain and eye abnormalities in addition to the muscular dystrophy. To our knowledge, three such families have been described in previous reports $[1,2,4]$. Due to the small number of cases described, little is known about the range of clinical variability of disorders caused by $P O M K$ mutations.

We report clinical, molecular, histological, neurophysiological and imaging data on a sib pair with limb-girdle muscular dystrophy-dystroglycanopathy type $\mathrm{C} 12$, caused by a novel compound heterozygous mutation in the POMK gene. The incidental finding of an elevated serum creatine kinase (CK) activity in the probands in infancy, which eventually led to the diagnosis, allowed for observation of the onset and the progression of the disease.

\section{Case report}

\subsection{Clinical phenotype}

The probands comprise a Finnish sib pair born to nonconsanguineous parents. The older sister was born at term with birth asphyxia due to placental abruption. She suffered a mild asphyxia-related kidney injury for which she was on follow-up. At 6 years of age, while being investigated for elevated liver enzymes, she presented with elevated CK values of 1000-4000 U/L. Her motor and cognitive developments as well as her growth were normal. She started walking at age 15 months. Her visual acuity was normal. She had unilateral high-frequency hearing loss (hearing threshold 65 decibel at 6000 and $8000 \mathrm{~Hz}$ ). In childhood she suffered from transient growing pains. Since school age she has experienced infrequent hip cramps triggered by hip flexion when doing for instance sit-ups, and by cramps in the neck region triggered by yawning. She has, at times, suffered from exercise-induced knee ache. Testing of muscle strength at 10 years of age using myometry showed muscle power slightly below average for hip adductors and abductors, as well as for knee extensors and ankle plantar flexors, as compared with normative values. On the contrary, muscle power in other arm and leg muscle groups were equal to or even stronger than average [5]. Subsequent evaluations showed muscle strength within normal limits.

Her electromyography (EMG) findings were normal, however, only one muscle (right gastrocnemius) was examined due to her fear of needles. Neurography of the median, tibial peroneal and sural nerves gave normal results. A muscle biopsy at 7 years of age was paraffin-embedded and showed normal findings. Echocardiography (followed up every two years starting from 7 years of age, with the latest evaluation done at 14 years of age) and spirometry showed no signs of cardiac or respiratory involvement. Magnetic resonance imaging (MRI; 1,5 T scanner) with T1-weighted and STIR images of muscles in shoulders, upper arms, thighs and pelvic region was normal, without signs of fatty replacement or edema (not shown).

The younger brother was born preterm by caesarean section due to placenta praevia. His motor and cognitive developments as well as his growth were unremarkable. He started walking at age 13 months. He had normal hearing and visual acuity. As a consequence of his sisterś incidentally detected CK elevation, his CK level was determined, showing markedly increased levels of up to $6800 \mathrm{U} / \mathrm{L}$. In childhood, he suffered from transient nocturnal growing pains. He also suffered from thigh stiffness and infrequent pain and cramps in the thighs and groins triggered by hip flexion. Like his sister, he experienced infrequent neck region cramps triggered by yawning. Muscle strength in his hip abductors and adductors as well as upper limbs was slightly weak, making it, at times, difficult for him to do sit-ups and arm push-ups.

His EMG was normal (neurography of tibial, peroneal and sural nerve, as well as myography of vastus lateralis, tibialis anterior and biceps). A muscle biopsy taken from the vastus lateralis muscle at age 6 showed moderate chronic myopathic changes, small groups of regenerating fibres, sparse inflammatory cell infiltrates, $\alpha$-dystroglycan deficiency in a majority of fibres with preserved expression in single, scattered fibres and normal merosin immunolabelling (Fig. 1). The major histocompatibility complex 1 (MHC1) protein was slightly up-regulated (not shown). Electrocardiography (ECG) showed isolated persistent deep Q-waves in leads V5-6 from the age of 5 years onwards. At the age of 7 years, the boy had a history of syncope of unknown etiology (electroencephalography, echocardiography and 24-h ECG were normal). Echocardiography was normal at initial evaluation at the age of 5 years but gradually turned pathological after the age of 8 years, revealing mild enlargement and borderline weakened function of the left ventricle by the age of 12 years. No cardiac MRI was done. The boy is on regular cardiac follow-up (echocardiography and 24-h ECG) every 6-24 months and receives no treatment. There were no signs of respiratory involvement. MRI findings (1,5 T, T1-weighted and STIR images) in the muscles of the shoulder region, upper arms, thighs and pelvic region were within normal limits, without signs of fatty replacement, although with minimal high signal changes in the right shoulder and left pelvic and thigh regions (not shown).

On clinical examination at age 13 and 10 years, respectively, both the sister and brother had calf hypertrophy (Fig. 2), mild lumbar lordosis and slightly winged scapulae, brisk tendon reflexes (interpreted as normal) in the lower extremities, weakened but positive tendon reflexes in the upper extremities, absent Babinski signs and negative Gowersśigns. They had no mirror movements, which have been related to $P O M K$ mutations in a previous report [3]. The brother had difficulty in walking on heels. Both sibs were physically active and considered themselves symptom-free and as physically fit as their peers. Brain MRI was not performed. 

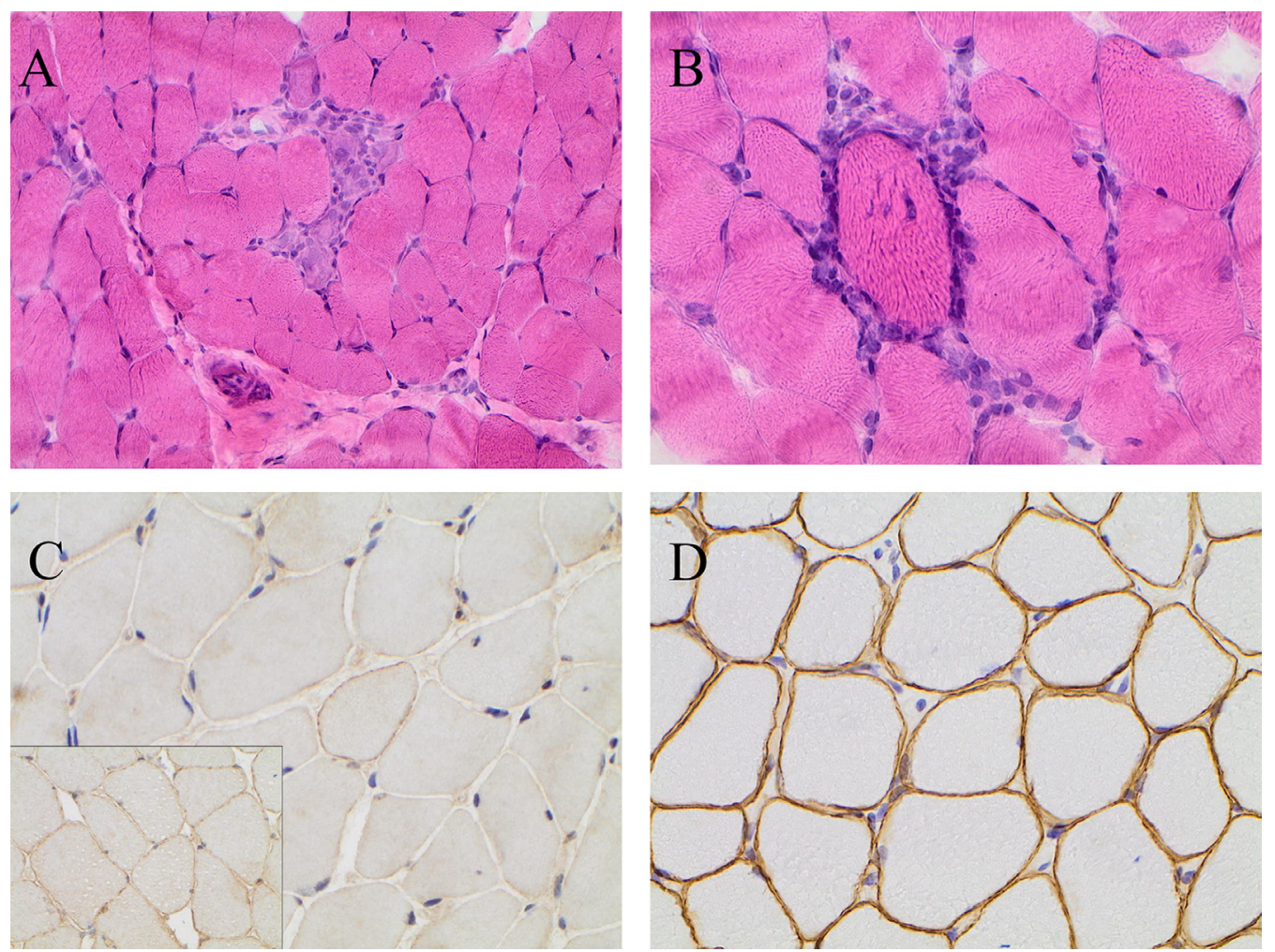

Fig. 1. Muscle biopsy of the brother demonstrating (a) small groups of regenerating fibres (Hematoxylin eosin, $\times 200$ ), (b) sparse inflammatory cell infiltrates (Hematoxylin eosin, $\times 400$ ), (c) weak alpha-dystroglycan expression with preserved staining in scattered single fibres (immunohistochemistry, alphadystroglycan, $\times 400$ ), (insert shows alpha-dystroglycan expression in a control muscle biopsy), and (d) normal merosin immunolabeling (immunohistochemistry, merosin, $\times 400)$.
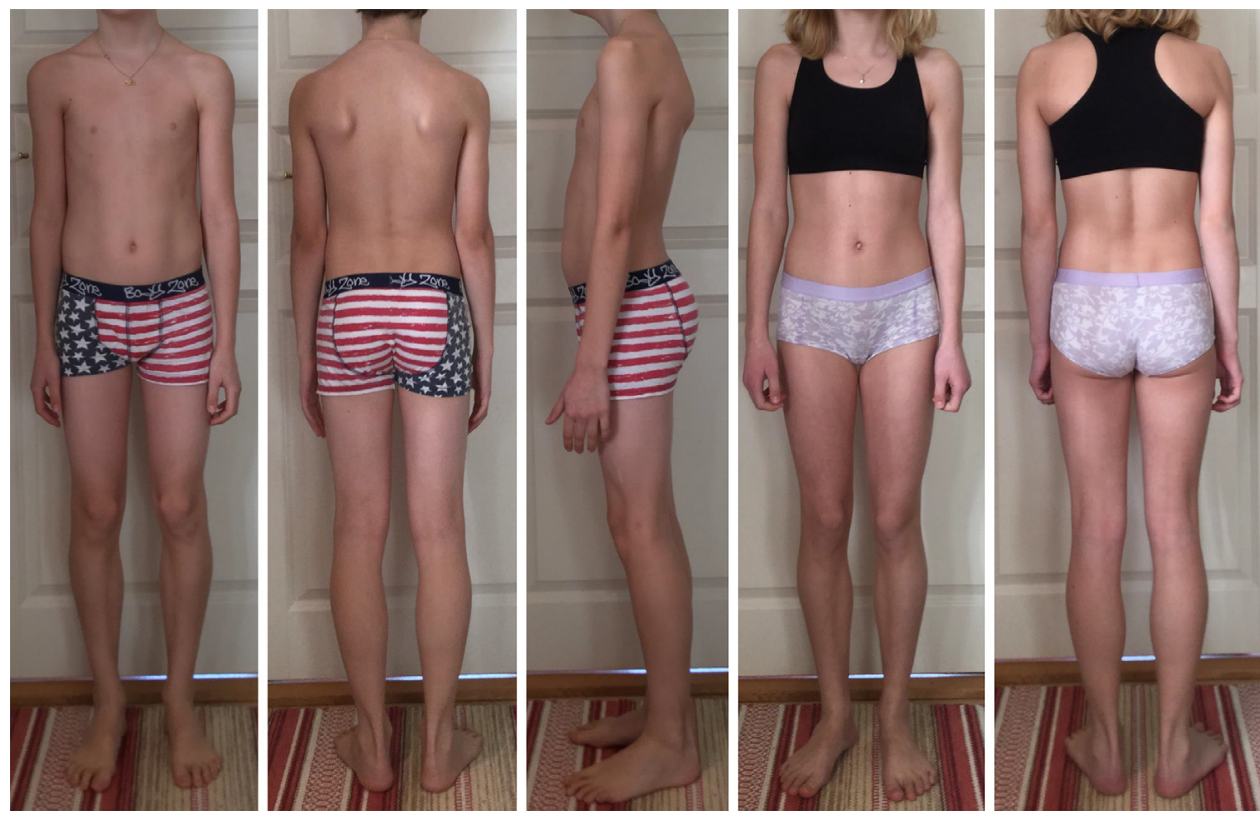

Fig. 2. Photographs of the probands showing calf hypertrophy, slightly accentuated lumbar lordosis and slightly winged scapulae. 
Family history of muscle disease was non-contributory, and the parents' $\mathrm{CK}$ levels were normal.

\subsection{Molecular findings}

The patients were enrolled into the MYO-SEQ project (myo-seq.org), an international research collaboration that applies whole exome sequencing (WES) to patients with undiagnosed limb-girdle muscle weakness. At least $250 \mathrm{ng}$ of DNA (>2 ng/ $\mu$ l) for each sibling was used as a template for WES. The resulting data were processed by the Genomics Platform at the Broad Institute of Harvard and MIT (Boston, MA, USA) as described previously [6]. The variant call set was uploaded onto the Broad Institute of Harvard and MIT's seqr platform (https://www.seqr.broadinstitute.org) and $P O M K$ was analysed for biologically relevant variants previously described by Johnson et al. [7].

Both siblings were shown to harbour two rare variants in POMK. The first was a stop-gained nonsense mutation, p.Arg46Ter (hg19 chr8:42958827, c.136C > T), and the second was a missense mutation, p.Pro322Leu (hg19 chr8:42977932, c.965C > T). Both variants are very rare in the control population (minor allele frequency $[\mathrm{MAF}]<0.0001)$ and have never been found in homozygosis. Sanger sequencing confirmed the presence of the variants in trans in the siblings. Each unaffected parent was only heterozygous for one of the variants each. We have submitted the variants and phenotype descriptions to a public database; the Leiden Open Variant Database (https://www.LOVD.nl).

\section{Discussion}

The POMK gene (synonym SGK196) encodes a 350 amino acid protein comprising 5 exons [4]. The full-length wildtype $P O M K$ protein has a predicted molecular weight of $40.15 \mathrm{kDa}$. The gene is conserved in mammals and amphibians [2]. Supporting the presumption that POMK mutations cause muscle disease, Di Costanzo and colleagues observed high expression of $P O M K$ in muscle and brain tissues during fetal development, especially during myocyte differentiation. In zebrafish embryos, knockdown of $P O M K$ led to muscular dystrophy, stressing the importance of $P O M K$ for early muscle development [1]. Moreover, protein analysis studies by von Renesse et al. showed an absence of POMK protein expression in fibroblasts and muscle cells derived from patients with homozygous truncating POMK mutations, whereas such expression was present in controls [2].

The novel mutations in our patients comprised one nonsense mutation (p.Arg46Ter) in exon 4, resulting in a premature termination of the amino acid sequence, and a missense mutation (p.Pro322Leu) in exon 5. High Combined Annotation-Dependent Depletion (CADD) scores of 35 and 27.7, respectively, suggest both variants are damaging to the protein [8]. Interestingly, all cases with POMK-related dystroglycanopathies reported to date have at least one mutation in exon 5 [1-4]. Despite the lack of functional studies of the specific mutations identified in our patients, the phenotype, consistent with LGMD, the elevated CK activity as well as the $\alpha$-dystroglycan deficiency in the muscle biopsy of the brother strongly argues for pathogenicity of the mutations. We interpret the slight up-regulation of MHC 1 as unspecific and compatible with the occurrence of occasional inflammatory infiltrates sometimes seen in muscle biopsies of patients with muscular dystrophy. Hence, the current report supports previous studies claiming a causative role of $P O M K$ mutations in dystroglycanopathies.

POMK-related dystroglycanopathies are ultra-rare autosomal recessive disorders. To our knowledge, when taking into account the current study, there are, to date, nine published cases from six different families [1-4]. As with dystroglycanopathies in general, their phenotypic presentations vary widely. On comparison with the published cases, we were unable to pinpoint any clear-cut genotype-phenotype correlation. Whether this lack of correlation is explained by chance due to the small sample, or by a factual lack of correlation, remains to be seen as new cases are identified in the future. Our two patients, who had a childhood onset of mild LGMD with normal cognition, represent the least severe phenotype among the published POMK cases. The mild cardiac involvement observed in the boy, however, has not been reported previously and may either represent a new phenotypic feature of $P O M K$-related dystroglycanopathies or be unrelated to it. The two Jordanian sibs with MDDGC12 had a clinical presentation of infancy-onset, fairly slowly progressive LGMD with cognitive deficit, caused by a severe protein-truncating homozygous missense mutation in POMK [1]. Interestingly, the same allelic variant was identified in a more severely affected Lebanese sib pair diagnosed with MDDGA12, who had infancy-onset muscle weakness and hypotonia, developmental delay, wheel-chair dependency, affected vision and brain MRI abnormalities - again illustrating the wide phenotypic variation [2]. Furthermore, the adolescent boy reported by Ardicli et al. had childhood-onset slowly progressive LGMD with congenital mirror movements and brain abnormalities on MRI, caused by a homozygous missense mutation in $P O M K$ [3]. Finally, an Italian boy diagnosed with MDDGA12 had a severe infancy-onset phenotype of hypotonia, cognitive impairment, epilepsy, and brain and eye abnormalities. He deceased at the age of 4 years and had a compound heterozygous POMK mutation; one frameshift mutation causing a premature termination and another mutation causing the substitution of a highly conserved amino acid in exon 5 [1].

In summary, we report novel compound heterozygous POMK mutations in two siblings with a childhood onset LGMD, who are mildly affected with a slow or nonprogressive disease course. They were cognitively unaffected and seemed to have isolated muscle affection, however, the cardiac pathology in the brother may constitute a new phenotypic feature of MDDGA12C. This report adds to the literature by providing new phenotypic data on this ultra-rare disease. As with rare diseases in general, further case reports are needed to elucidate the entire phenotypic spectrum of POMKrelated dystroglycanopathies. 


\section{Acknowledgements}

We are grateful to the family who gave their permission to publish data and photographies. This study was financially supported by the Sigrid Jusélius Foundation, the Association Francaise contre les Myopathies, the Finska Läkaresällskapet, Muscular Dystrophy UK and the Medicinska understödsföreningen Liv och Hälsa. The MYO-SEQ study was funded by Sanofi Genzyme, Ultragenyx, Kurt + Peter Foundation, LGMD2I Research Fund, Samantha J Brazzo Foundation, LGMD2D Foundation, Muscular Dystrophy UK and Coalition to Cure Calpain 3. The companies, foundations and charities were not involvement in the study design, data acquisition, data analysis, manuscript preparation or manuscript revision.

\section{References}

[1] Di Costanzo S, Balasubramanian A, Pond HL, Rozkalne A, Pantaleoni C, Saredi S, et al. POMK mutations disrupt muscle development leading to a spectrum of neuromuscular presentations. Hum Mol Genet 2014;23(21):5781-92.
[2] von Renesse A, Petkova MV, Lützkendorf S, Heinemeyer J, Gill E, Hübner $\mathrm{C}$, et al. $P O M K$ mutation in a family with congenital muscular dystrophy with merosin deficiency, hypomyelination, mild hearing deficit and intellectual disability. J Med Genet 2014;51:275-82.

[3] Ardicli D, Gocmen R, Talim B, Sprute R, Haliloglu G, Cirak S, et al. Congenital mirror movements in a patient with alpha-dystroglycanopathy due to a novel POMK mutation. Neuromusc Disord 2017;27(3):239-42.

[4] Jae LT, Raaben M, Riemersma M, van Beusekom E, Blomen VA, Velds A, et al. Deciphering the glycosylome of dystroglycanopathies using haploid screens for lassa virus entry. Science 2013;340(6131):479-83.

[5] Eek MN, Kroksmark AK, Beckung E. Isometric muscle torque in children 5 to 15 years of age: normative data. Arch Phys Med Rehabil 2006;87(8):1091-9.

[6] Perić S, Glumac JN, Töpf A, Savić-Pavićević D, Phillips L, Johnson $\mathrm{K}$, et al. A novel recessive TTN founder variant is a common cause of distal myopathy in the Serbian population. Eur J Hum Genet 2017;25(5):572-81.

[7] Johnson K, Töpf A, Bertoli M, Phillips L, Claeys KG, Stojanovic VR, et al. Identification of GAA variants through whole exome sequencing targeted to a cohort of 606 patients with unexplained limb-girdle muscle weakness. Orphanet J Rare Dis 2017;12(1):173.

[8] Kircher M, Witten DM, Jain P, O'Roak BJ, Cooper GM, Shendure J. A general framework for estimating the relative pathogenicity of human genetic variants. Nat Genet 2014;46(3):310-15. 